\title{
Leukemia cells remodel marrow adipocytes via TRPV4-dependent lipolysis
}

Haematologica 2020

Volume 105(11):2572-2583

\section{Correspondence:}

JUN SHI

junshi@sjtu.edu.cn

ZHIQIANG LI

kcb039@126.com

Received: April 30, 2019.

Accepted: December 18, 2019.

Pre-published: December 19, 2019.

doi:10.3324/haematol.2019.225763

(C)2020 Ferrata Storti Foundation

Material published in Haematologica is covered by copyright. All rights are reserved to the Ferrata Storti Foundation. Use of published material is allowed under the following terms and conditions:

https://creativecommons.org/licenses/by-nc/4.0/legalcode. Copies of published material are allowed for personal or internal use. Sharing published material for non-commercial purposes is subject to the following conditions:

https://creativecommons. orǵ/licenses/by-nc/4.0/legalcode, sect. 3. Reproducing and sharing published material for commercial purposes is not allowed without permission in writing from the publisher.

\author{
Shaoxin Yang,,$^{1^{*}}$ Wei Lu, ${ }^{1 *}$ Chong Zhao, ${ }^{2}$ Yuanmei Zhai, ${ }^{3}$ Yanyu Wei, ${ }^{1}$ \\ Jiali Liu, ${ }^{2}$ Yehua $\mathrm{Yu},{ }^{1}$ Zhiqiang $\mathrm{Li}^{4}$ and Jun $\mathrm{Shi}^{1}$
}

${ }^{1}$ Department of Hematology, Shanghai Ninth People's Hospital, Shanghai JiaoTong University School of Medicine; '²Department of Hematology, Shanghai Jiao Tong University Affiliated Sixth People's Hospital; ${ }^{3}$ Department of Hematology, Tongren Hospital, Shanghai Jiao Tong University School of Medicine and ${ }^{4}$ Department of Blood Transfusion, Shanghai Jiao Tong University Affiliated Sixth People's Hospital, Shanghai, China.

*SY and WL contributed equally as co-first authors.

\section{ABSTRACT}

$\mathrm{R}$ emodeling of adipocyte morphology and function plays a critical role in prostate cancer development. We previously reported that leukemia cells secrete growth differentiation factor 15 (GDF15), which remodels the residual bone marrow (BM) adipocytes into small adipocytes and is associated with a poor prognosis in patients with acute myeloid leukemia. However, little is known about how GDF15 drives BM adipocyte remodeling. In this study, we examined the role of the transient receptor potential vanilloid (TRPV) channels in the remodeling of BM adipocytes exposed to GDF15. We found that TRPV4 negatively regulated GDF15-induced remodeling of BM adipocytes. Furthermore, transforming growth factor- $\beta$ type II receptor was identified as the main receptor for GDF15 on BM adipocytes. PI3K inhibitor treatment reduced GDF15induced pAKT, identifying PIBK/AKT as the downstream stress response pathway. Subsequently, GDF15 reduced the expression of the transcription factor Forkhead box C1 (FOXC1) in BM adipocytes subjected to RNAsequencing screening and western blot analysis. Moreover, it was also confirmed that FOXC1 combined with the TRPV4 promoter by chromatin immunoprecipitation with quantitative polymerase chain reaction experiments, which suggests that FOXC1 mediates GDF15 regulation of TRPV4. In addition, an acute myeloid leukemia mouse model exhibited smaller BM adipocytes, whereas the TRPV4 activator $4 \alpha$-phorbol 12,13-didecanoate partly rescued this process and increased survival. In conclusion, TRPV4 plays a critical role in BM adipocyte remodeling induced by leukemia cells, suggesting that targeting TRPV4 may constitute a novel strategy for acute myeloid leukemia therapy.

\section{Introduction}

The development of acute myeloid leukemia (AML) is closely related to the bone marrow (BM) microenvironment. ${ }^{1,2}$ As a critical component of the BM microenvironment, BM adipocytes provide energy for both the infinite proliferation of leukemia cells and the normal growth of hematopoietic stem cells. ${ }^{3,4}$ Leukemia cells proliferate to an overwhelming number in a limited marrow cavity, likely because these cells are more efficient in capturing energy for growth. Accordingly, BM adipocytes are remodeled in response to leukemia cells, generating a pro-tumoral microenvironment. ${ }^{5,6}$ However, the mechanism whereby leukemia cell growth induces BM adipocyte remodeling is still unclear.

Induced BM adipocyte remodeling involves several specific processes, including lipolysis, dedifferentiation and lipid accumulation. Consequently, the remodeled adipocytes show morphological and functional changes.' Breast cancer cells reportedly secrete soluble factor Wnt3a which reduces the number and size of adipocytes surrounding the malignant cells and thus contributes to disease devel- 
opment. ${ }^{8}$ As the breast cancer progresses, adipocytes dedifferentiate to fibroblast-like cells. ${ }^{8}$ In mouse models of bone metastasis following prostate cancer, Herroon et al. showed that remodeled BM adipocytes support tumor growth by fatty acid-binding protein 4 (FABP4) transportation of fatty acids. ${ }^{9}$ These studies identified a functional role of remodeled adipocytes in supporting solid tumor metabolism. Thus, the adipocytes remodeled by cancer cells are also known as cancer-associated adipocytes. ${ }^{10}$ In the context of leukemia, there is a growing consensus that reduction in BM adipocyte number, once believed to be merely due to mechanical squeezing by the rapid proliferation of leukemia cells in the limited BM cavity, is also actively regulated by leukemia cells., s.11 $^{5}$ Indeed, we previously reported that growth differentiation factor 15 (GDF15) derived from leukemia cells regulates $\mathrm{BM}$ adipocyte remodeling by enhancing lipolysis. ${ }^{12}$ However, how extracellular GDF15 induces lipolysis within BM adipocytes remains elusive.

It has been reported that GDF15 enhances intracellular $\mathrm{Ca}^{2+}$ by increasing calcium voltage-gated channel subunit alpha1 C (Cav1.3) expression in rat cerebellar granule neurons, which induces the expression of genes essential for synaptic plasticity. ${ }^{13}$ As an important cellular signal for lipid metabolism, intracellular $\mathrm{Ca}^{2+}$ is involved in lipid synthesis and lipolysis in adipocytes. ${ }^{14,15}$ When the calcium channels in the adipocytes are activated or upregulated, accumulation of lipids is enhanced through increased $\left[\mathrm{Ca}^{2+}\right]_{\mathrm{i}}{ }^{16,17}$ Conversely, when calcium channels are inhibited or downregulated, decreased calcium influx may accelerate fat breakdown. ${ }^{18,19}$ Thus, we hypothesized that calcium channels are involved in GDF15-induced BM adipocyte remodeling.

In this study, we examined a possible role of transient receptor potential vanilloid 4 (TRPV4) calcium channels in GDF15-driven remodeling of BM adipocytes. We unravel a novel function of transforming growth factor- $\beta$ type II receptor (TGFBRII) that, in responding to GDF15 in BM adipocytes, activates the phosphatidylinositol 3kinase (PIBK)/AKT transduction pathway, which in turn reduces the transcript factor Forkhead box C1 (FOXC1) level and subsequently downregulates TRPV4. We also provide evidence that inhibition of BM adipocyte remodeling increases survival in the AML mouse model, implying a novel therapeutic target for AML.

\section{Methods}

\section{Patients' samples}

BM aspirates were collected from 16 patients diagnosed as having lymphoma without BM invasion, using procedures approved by the Ethics Committee of Shanghai Jiao Tong University Affiliated Hospital. Mesenchymal stem cells were derived from the BM of lymphoma patients without BM invasion, because marrow mesenchymal stem cells in this type of patients can be considered normal. The adipogenic induction of mesenchymal stem cells is described in the Online Supplementary Methods.

\section{Chromatin immunoprecipitation-quantitative polymerase chain reaction}

Adipocytes were collected from different groups and crosslinked with $1 \%$ formaldehyde for $10 \mathrm{~min}$ at $37^{\circ} \mathrm{C}$. Crosslinking was blocked, then the cells were washed and lysed in sodium dodecylsulfate lysis buffer ( $50 \mathrm{mM}$ HEPE NaOH 7.5, 500 $\mathrm{mM} \mathrm{NaCl}, 1 \mathrm{mM}$ EDTA, $0.1 \%$ Na-deoxycholate, $1 \%$ Triton X100). The lysates were sonicated to shear DNA to a length between 200 and 500 base pairs with 10-second pulses using sonication. The antibody against FOXC1 (5 $\mu \mathrm{L}$, Abcam5079, USA) was then added to the supernatant, incubated overnight at $4^{\circ} \mathrm{C}$ with rotation and incubated with $100 \mu \mathrm{L}$ Salmon Sperm DNA/Protein A agarose beads for $2 \mathrm{~h}$ at $4^{\circ} \mathrm{C}$. The immunoprecipitated complex was then washed and eluted. The histone DNA crosslinks were reversed and DNA was purified for real-time polymerase chain reaction (PCR). Quantitative real-time PCR (RTqPCR) was performed on bound and input DNA with the following primers for TRPV4: forward: 5-CTTTGCACTGGGGAGCAGAGT-3, reverse: 5-ATTAACCG TGGGCTTCAGGCA-3.

\section{Cell cultures and reagents}

The cell cultures and reagents, as well as the co-culture assays are described in detail in the Online Supplementary Methods.

\section{Animal experiments}

All animal experiments were performed according to procedures approved by the Ethics Committee of Shanghai Jiao Tong University Affiliated Hospital. Five-week old C57BL/6 mice were fed with 60\% high-fat diet (Research Diets, Inc. New Brunswick, NJ, USA) for 3 months to create an obese mouse model. Mice injected with FBL-3 cells $\left(5 \times 10^{5}\right)$ and mice injected with both FBL-3 cells $\left(5 \times 10^{5}\right)$ and $4 \alpha$-phorbol 12,13-didecanoate (4aPDD) $(200 \mu \mathrm{g} / \mathrm{Kg}$ according to the instructions for reagents) were used as experimental groups. The untreated obese mice were used as a control group. The volume of all solutions injected was $200 \mu \mathrm{L}$. Mice were sacrificed and femora were removed after 3 weeks of treatment. Femora were fixed for $24 \mathrm{~h}$ with $4 \%$ paraformaldehyde and were decalcified for 2 days. BM sections from the mice were dewaxed by conventional methods and incubated with anti-perilipin1 monoclonal antibody (1:50, CST, USA) at $4^{\circ} \mathrm{C}$ overnight.

\section{Other experimental details}

Full descriptions of free fatty acid detection, lentiviral knockdown, RNA sequencing, western blot analysis and enzymelinked immunosorbent assays, RT-qPCR (primers shown in Table 1), the cell counting and apoptosis assays, oil red $\mathrm{O}$ staining, immunofluorescence studies, and adipocyte measurements are provided in the Online Supplementary Methods.

\section{Statistical analysis}

All statistical tests were performed with GraphPad Primer5. The data are presented as the mean \pm standard deviation. A Student $t$-test was used for comparisons between two groups. A $P$ value of less than 0.05 was considered statistically significant.

\section{Results}

\section{Downregulated TRPV4 contributes to increased bone marrow adipocyte lipolysis}

As an important channel for calcium ions, TRPV plays a critical role in the energy balance of adipocytes. RTqPCR analysis showed that TRPV4 mRNA in BM adipocytes had the highest expression among TRPV family members (Figure 1A). Moreover, western blot analysis showed that BM adipocytes expressed TRPV4 protein highly (Figure 1B). To investigate whether TRPV4 plays an important role in BM adipocytes, we used TRPV4 inhibitor (RN1734) and agonist (4aPDD) to verify the 
A

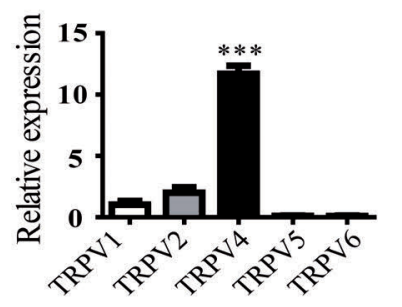

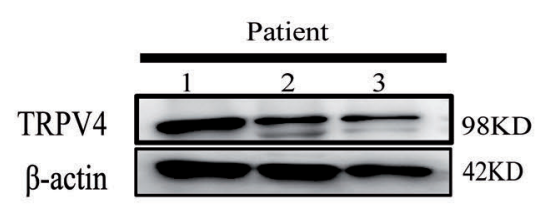

C

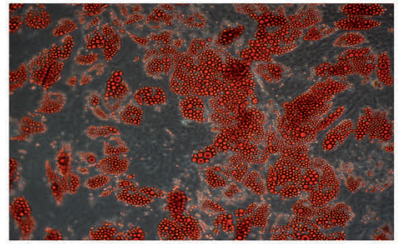

Ctr

D

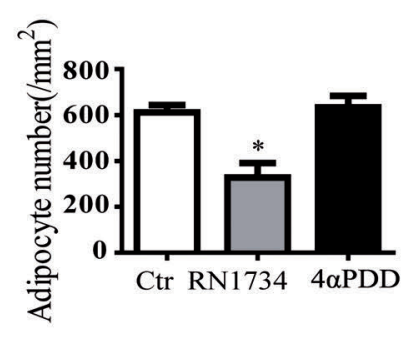

F

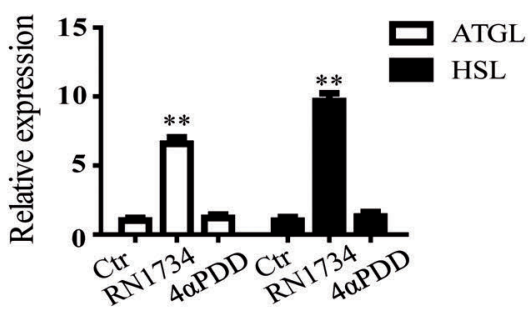

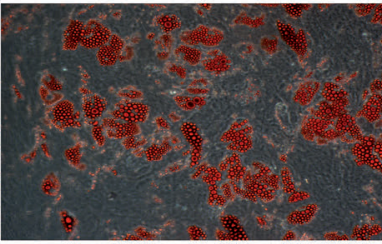

RN1734

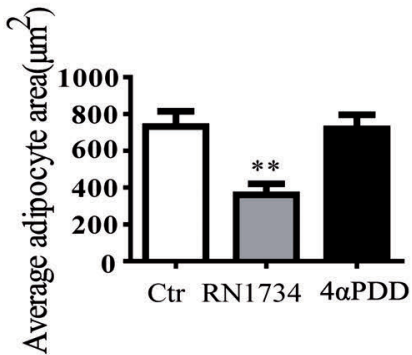

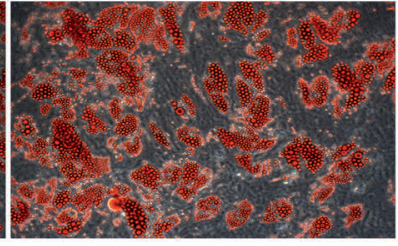

$4 \alpha \mathrm{PDD}$

E

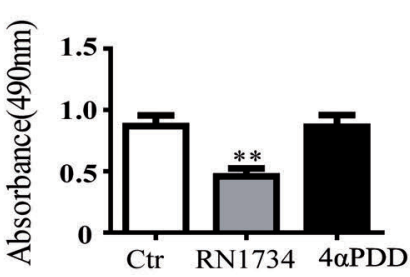

G

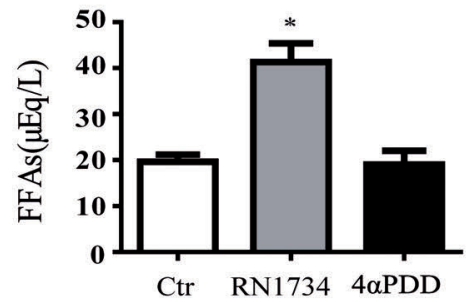

H

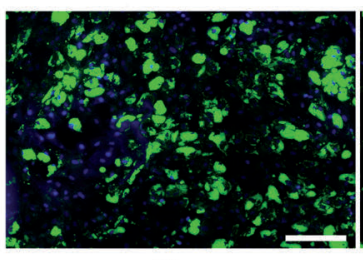

Ctr

I

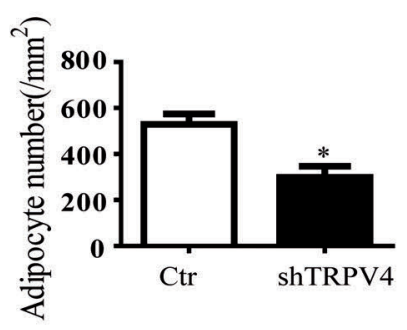

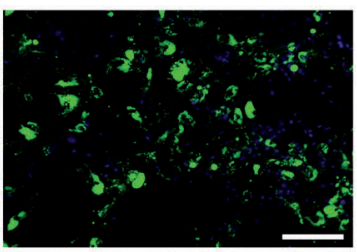

shTRPV4

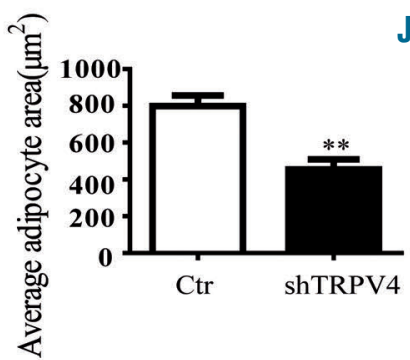

J

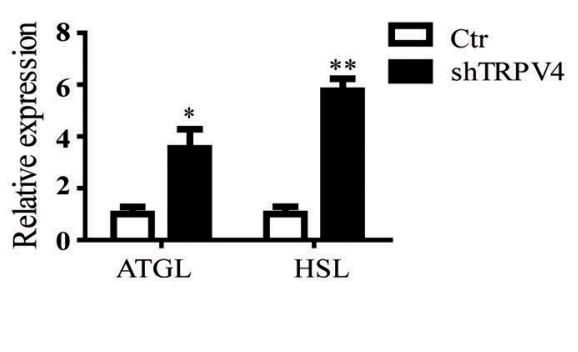

Figure 1. Downregulated TRPV4 contributes to increased bone marrow adipocyte lipolysis. (A) Reverse transcriptase quantitative polymerase chain reaction (RTGPCR) verification of the expression of transient receptor potential vanilloid (TRPV) channel genes in bone marrow (BM) adipocytes. (B) Western blot analysis of TRPV4 protein in BM adipocytes from three patients. (C) BM adipocytes treated with dimethylsulfoxide (Ctr), RN1734 (5 $\mu$ M) or $4 \alpha$-phorbol $12,13-$ didecanoate $(4 \alpha \mathrm{PDD}, 0.25 \mu \mathrm{g} / \mathrm{mL}$ ) for 4 days. Adipocytes were stained by oil red O (ORO). All images were at a magnification of 200×. (D) The number and average area of BM adipocytes from the indicated groups were measured using Image-Pro-Plus 5.1. (E) The content of lipid droplets in BM adipocytes from the indicated groups was detected by optical density values after ORO staining. (F) RT-qPCR was used to analyze adipose triglyceride lipase (ATGL) and hormone-sensitive triglyceride lipase (HSL) mRNA in BM adipocytes from the indicated groups. (G) The content of free fatty acids (FFA) in the supernatant of BM adipocytes treated with dimethylsulfoxide (Ctr), RN1734 or 4 RPDD was detected using the colorimetric method. (H) BM adipocytes were infected with TRPV4-targeted shRNA (shTRPV4) lentivirus for 6 days. Adipocytes were stained with Alexa Fluor 493/503-conjugated BODIPY. 4',6-diamidino-2-phenylindole (DAPI) stained blue and lipid droplets showed green fluorescence. The scale bar represents $50 \mu \mathrm{m}$. (I) The number and area of BM adipocytes infected with shTRPV4 lentivirus, quantitatively analyzed by Image-Pro-Plus 5.1 . $(J)$ The mRNA level of HSL and ATGL in BM adipocytes infected with shTRPV4 lentivirus on the fourth day, detected by RT-qPCR. $\beta$-actin protein was used as an internal control for the western blot analysis. Three independent experiments were performed. ${ }^{*} * P<0.001, * * P<0.01, * P<0.05$. 
function of TRPV4 in BM adipocytes, respectively. $4 \alpha \mathrm{PDD}$ is the first synthetic TRPV4 agonist and is a nonprotein kinase $\mathrm{C}$ activated phorbol ester. ${ }^{20}$ Online Supplementary Figure S1A shows the half maximal inhibitory concentration $\left(\mathrm{IC}_{50}\right)$ of the effects of RN1734 on $\mathrm{BM}$ adipocytes. Considering excessive $\mathrm{Ca}^{2+}$ influx could cause some toxicity to adipocytes, ${ }^{21}$ we aimed to find a concentration that has minimum cellular toxicity and promotes the $\mathrm{Ca}^{2+}$ influx needed for our experiments. As Online Supplementary Figure S1B shows, $4 \alpha \mathrm{PDD}$ at a concentration of $0.25 \mu \mathrm{g} / \mathrm{mL}$ resulted in an acceptable level of toxicity of adipocytes, while allowing $\mathrm{Ca}^{2+}$ influx to reach the level required for the experiment. Oil red $\mathrm{O}$ staining and quantitative analysis showed that RN1734 reduced the number and area of BM adipocytes, whereas $4 \alpha \mathrm{PDD}$ did not induce a similar change (Figure 1C, D), suggesting that the inhibition of TRPV4 contributes to reducing BM adipocyte number and size. Furthermore, optical density value measurements showed that lipid droplets in BM adipocytes treated with RN1734 decreased significantly (Figure 1E).

In order to determine whether the phenomenon is related to lipolysis, we determined the rate-limiting enzymes (adipose triglyceride lipase, ATGL and hormone sensitive lipase, HSL) of lipolysis. ${ }^{22}$ ATGL catalyzes the first step of lipolysis and converts triglyceride to diacylglycerol and free fatty acids. ${ }^{23} \mathrm{HSL}$ is a hydrolase of glycerides and cholesterol esters. ${ }^{24}$ Along with TRPV4 channel inhibition, BM adipocytes subsequently exhibited increased expression of $A T G L$ and $H S L$, which resulted in increased free fatty acids in the supernatant (Figure 1F, G). Furthermore, it was found that RN1734 could significantly inhibit $\mathrm{Ca}^{2+}$ influx in $\mathrm{BM}$ adipocytes, while $4 \alpha \mathrm{PDD}$ promoted $\mathrm{Ca}^{2+}$ influx in $\mathrm{BM}$ adipocytes (Online Supplementary Figure S1C). However, $4 \alpha \mathrm{PDD}$ activates calcium channels in BM adipocytes by promoting $\mathrm{Ca}^{2+}$ influx, while the expression of TRPV4 could not increase (Online Supplementary Figure S2A).

To further confirm that TRPV4 regulates lipolysis of BM adipocytes, we used shTRPV4 lentivirus to knock down TRPV4 (Online Supplementary Figure S2B, C). As shown in Figure $1 \mathrm{H}, \mathrm{I}$ and Online Supplementary Figure $S 2 D$, quantitative analysis showed that the number (control vs. shTRPV4, $528.1 \pm 46.4 / \mathrm{mm}^{2}$ vs. $298.9 \pm 48.3 / \mathrm{mm}^{2}$, $P<0.05$ ) and area (control vs. shTRPV4, 798.7 $\pm 57.5 u^{2} \mathrm{~m}^{2}$ vs. $\left.454.7 \pm 54.0 \mu^{2}, P<0.01\right)$ of BM adipocytes decreased in TRPV4 knockdown samples. ATGL and HSL mRNA levels were also increased in TRPV4 knockdown adipocytes (Figure 1J). These data indicate a critical role for TRPV4 in the regulation of lipolysis in BM adipocytes.

\section{TRPV4 mediates GDF15-induced bone marrow adipocyte remodeling}

Increased lipolysis can result in a decrease in the number and area of BM adipocytes. Therefore, lipolysis is also a form of adipocyte remodeling. Our previous studies found that GDF15 secreted by leukemia cells promoted $\mathrm{BM}$ adipocyte lipolysis, decreasing the number and area of BM adipocytes. ${ }^{5,12}$ As shown by western blot analysis, TRPV4 expression was inhibited in BM adipocytes when co-cultured with leukemia cell lines (THP-1, K562, HL60), whereas anti-GDF15 neutralizing antibodies partly reversed the effect (Figure 2A). Given the above results, we added recombinant human GDF15 (rhGDF15) to BM adipocytes to clarify this effect. It was shown that the inhibitory effect on TRPV4 was enhanced with the increase of rhGDF15 concentration and treatment duration (Figure 2B, C). Moreover, rhGDF15 could significantly inhibit TRPV4 mRNA expression and increase pHSL protein expression on the fourth day (Figure 2C and Online Supplementary Figure S2E). However, the pHSL protein and the release of free fatty acids did not increase sig-

Table 1. Sequences of the primers used to detect gene expression by reverse transcriptase quantitative polymerase chain reaction.

\begin{tabular}{clll} 
Species & Name & Forward & Reverse \\
human & GAPDH & CGGAGTCAACGGATTTGGTCGTAT & AGCCTTCTCCATGGTGGTGAAGAC \\
human & TRPV1 & CAGGCTCTATGATCGCAGGAG & TTTGAACTCGTTGTCTGTGAGG \\
\hline human & TRPV4 & CGTCCAAACCTGCGAATGAAGTTC & CCTCCATCTCTTGTTGTCACTG \\
human & TRPV5 & GGTTTTCTACCTAAGGCAGAAGG & CTCGAAGCAGTGGAGACTCT \\
\hline human & TRPV6 & ACTGACCTCGACTCTCTATGAC & GTGGTGATGATAAGTTCCAGCAG \\
human & ALK4 & GCCATGGGAAGTTGTAATGG & GTCCAGGTGCCATTATTCAG \\
human & ACVR2 & GCCACCCTATTACAACATCCTG & GGTCCTGGGTCTTGAGTTG \\
human & TGFßRI & GAAGAGGACCCTTCATTAG & TGCCTCACGGAACCACGAACG \\
human & TGFßRII & CAACAACATCAACCACAACA & TTATAGACCTCAGCAAAGCG \\
\hline human & GFRAL & ATGGATTCAAAGGGATGTG & TGATAGAAGAACCGTATGGC \\
human & ATGL & GCGTGTCAGACGGCGAGAATG & GCAGCTCGTGGATGTTGGTGG \\
\hline human & HSL & CACTACAAACGCAACGAGAC & CCAGAGACGATAGCACTTCC \\
human & FOXCl & GCCGCACAGTCCCATCTCT \\
\hline human & Cav1.3 & TAAGCCCATGAATCAGCCG & TCATACATCACCGCATTCC \\
human & Cav3.1 & CTTCCTCTTCATCATCATCTTC & GGGTCAGGAAGATGCGTTCA \\
\hline human & Cav3.2 & CCACGTGGTCCTTGTCATCA & TGCATCCAGGAATGGTGAG \\
\hline
\end{tabular}

GAPDH: glyceraldehyde 3-phosphate dehydrogenase;TRPV: transient receptor potential vanilloid;ALK4: activin A receptor type 1B; ACVR2: activin receptor type 2;TGFßRII: transforming growth factor- $\beta$ type II receptor; TGF $\beta$ RI: transforming growth factor- $\beta$ type I receptor; GFRAL: GDNF family receptor $\alpha$-like; ATGL: adipose triglyceride lipase; HSL: hormone-sensitive triglyceride lipase; FOXC1: Forkhead box C1; Cav1.3: calcium voltage-gated channel subunit alpha1 C; Cav3.1: calcium voltage-gated channel subunit alpha1 G; Cav3.2: calcium voltage-gated channel subunit alpha1 $\mathrm{H}$; Cav3.1: calcium voltage-gated channel subunit alpha1 I. 
A

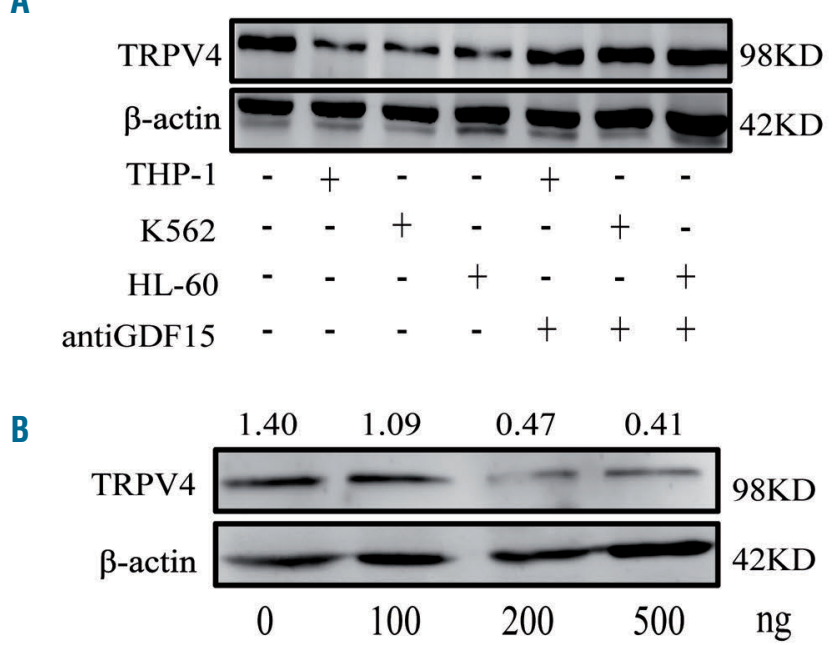

C

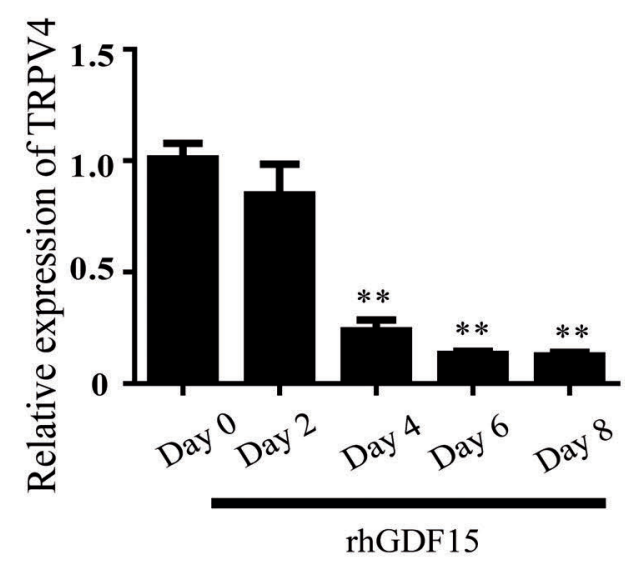

D
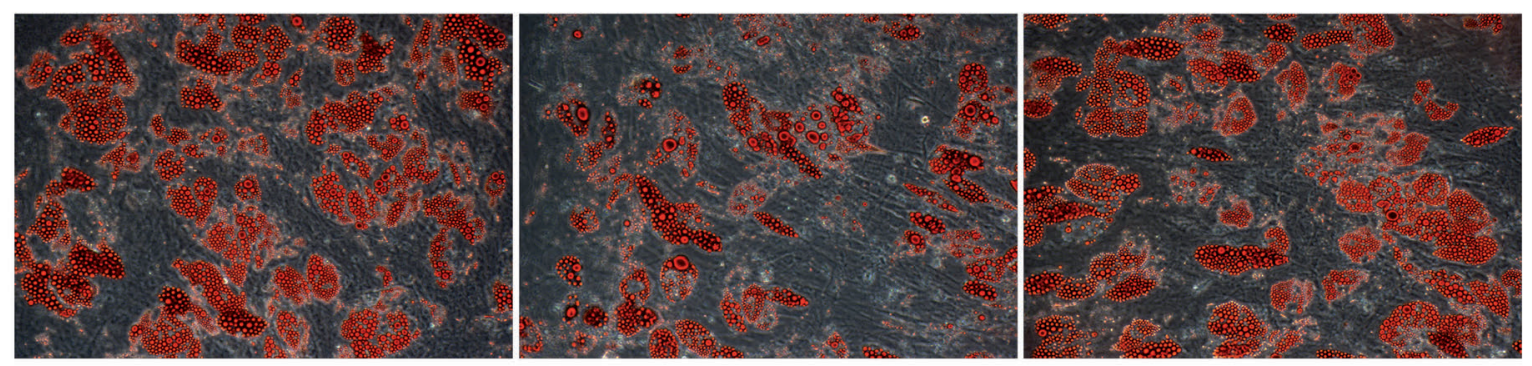

$\mathrm{E}$
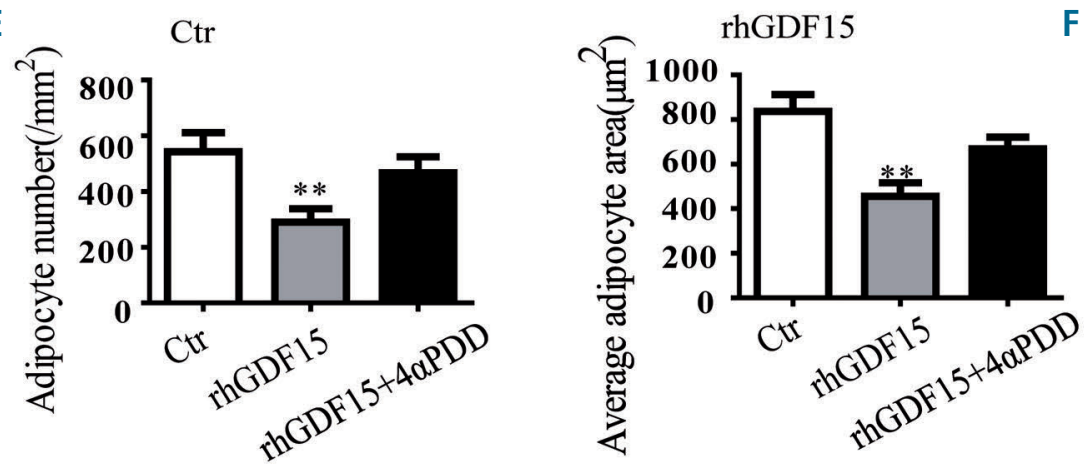

rhGDF15 $+4 \alpha \mathrm{PDD}$

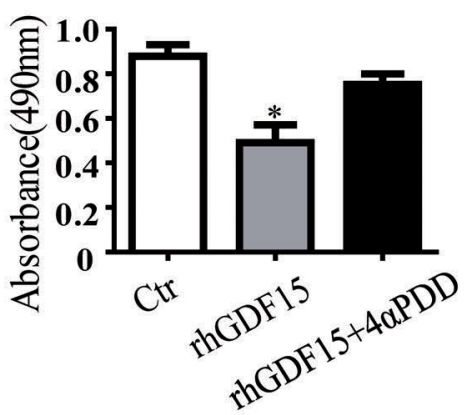

G

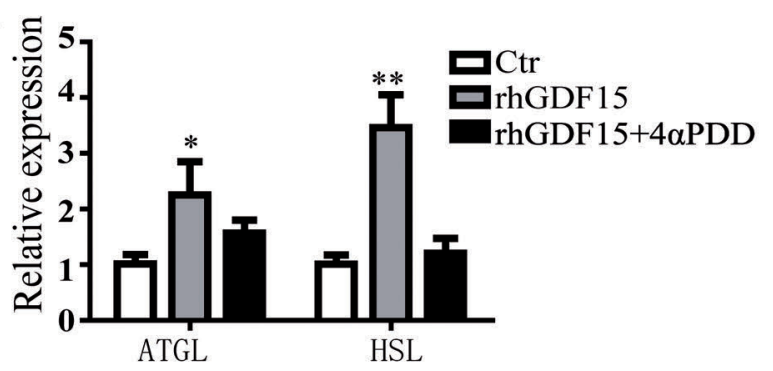

H

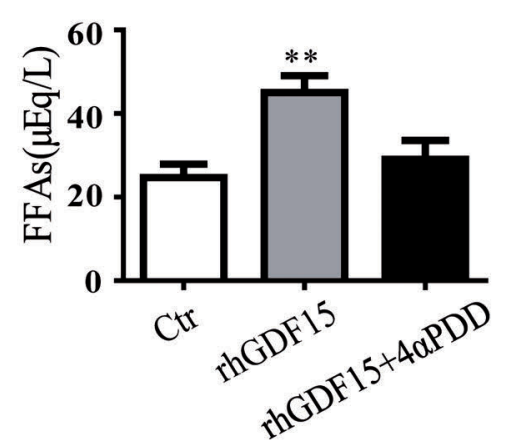

Figure 2. TRPV4 mediates GDF15-induced bone marrow adipocyte remodeling. (A) Bone marrow (BM) adipocytes were co-cultured with leukemia cell lines (THP-1, K562, HL-60) or leukemia cells and anti-GDF15 neutralizing antibody (200 ng/mL) for 4 days. The protein of TRPV4 was detected using western blot analysis. (B) The effect of different concentrations ( $100 \mathrm{ng}, 200 \mathrm{ng}, 500 \mathrm{ng}$ ) of recombinant human GDF15 (rhGDF15) on the expression of TRPV4 protein for 4 days was analyzed by western blot. (C) Reverse transcriptase quantitative polymerase chain reaction (RT-qPCR) was performed to analyze the expression of TRPV4 mRNA after the addition of 200 ng rhGDF15 in BM adipocytes for 2, 4, 6, 8 days. (D) BM adipocytes were treated with dimethylsulfoxide (Ctr), rhGDF15 (200 ng/mL) or rhGDF15 (200 $\mathrm{ng} / \mathrm{mL}$ ) and $4 \alpha$-phorbol 12,13-didecanoate $(4 \alpha P D D, 0.25 \mu \mathrm{g} / \mathrm{mL}$ ) for 6 days. Adipocytes were stained by oil red 0 . All images were at a magnification of $200 \times$. (E) The number and average area of adipocytes from the indicated groups were measured using Image-Pro-Plus 5.1. (F) The content of lipid droplets in the indicated groups was detected by optical density values. (G) RT-qPCR was used to analyze HSL and ATGL mRNA in adipocytes from the indicated groups on the fourth day. (H) The content of free fatty acids in the supernatant of BM adipocytes from each group was detected using a colorimetric method. $\beta$-actin protein was used as an internal control for the western blot analysis. Three independent experiments were performed. $* * P<0.01, * P<0.05$. 
nificantly after BM adipocytes were treated with rhGDF15 for $1 \mathrm{~h}$ (Online Supplementary Figure S2F, G), suggesting that the role of GDF15 in promoting lipolysis may be different from the rapid action of $\beta$-adrenaline. ${ }^{25}$ Oil red $\bigcirc$ staining and quantitative analysis showed that the number and area of adipocytes did not change significantly on the fourth day, but decreased significantly from the sixth day (Online Supplementary Figure S2H, I). These results indicate that TRPV4 regulates the remodeling of BM adipocytes.

To further explore the potential role of TRPV4 in GDF15-induced BM adipocyte remodeling, oil red $O$ staining and quantitative analysis was conducted and showed that the number and area of BM adipocytes were decreased in BM adipocytes treated with rhGDF15, whereas $4 \alpha \mathrm{PDD}$ partly reversed the effect of rhGDF15 (Figure 2D, E). Optical density value measurements of lipid droplets showed similar results (Figure 2F). Accordingly, rhGDF15 could induce increased expression of lipolysis genes (ATGL and HSL) and increased release of free fatty acids from BM adipocytes, but activation of TRPV4 by $4 \alpha$ PDD partly reversed the effect of rhGDF15 (Figure 2G, H). Furthermore, rhGDF15 can inhibit $\mathrm{Ca}^{2+}$ influx in BM adipocytes (Online Supplementary Figure $S 3 A$ ). These findings strongly suggest that TRPV4 contributes to GDF15-induced remodeling of BM adipocytes. Although GDF15 has been reported to act on Cav1.3, Cav3.1, Cav3.2, Cav3.3, ${ }^{13,26}$ the expression of these channels in BM adipocytes is much lower than that of TRPV4 (Online Supplementary Figure S3B). Moreover, when BM adipocytes were co-cultured with leukemia cell lines (THP-1, K562, HL-60), the expression of TRPV4 changed significantly (Online Supplementary Figure S3C). These results further suggest that TRPV4 may play an important role in GDF15-induced remodeling of BM adipocytes.

\section{GDF15 activates the downstream genes PI3K and pAKT in bone marrow adipocyte remodeling}

Extracellular GDF15 must bind to a receptor on the membrane surface to cause intracellular changes in BM adipocytes. We screened all of the reported GDF15 receptors by RT-qPCR and found that BM adipocytes mainly express TGF $\beta$ RI and TGF $\beta$ RII (Figure $3 \mathrm{~A}$ ). In order to determine whether GDF15 acts through binding to TGF $\beta$ RI or TGF $\beta$ RII on BM adipocytes, we conducted inhibitor experiments in vitro. ${ }^{27,28}$ Western blot results showed that rhGDF15 could downregulate TRPV4 expression in BM adipocytes treated with RepSox (a TGF $\beta R$ I inhibitor) rather than ITD1 (a TGFßRII inhibitor) (Figure 3B). Moreover, we found that rhGDF15 could reduce the number and area of BM adipocytes treated with RepSox as compared to those treated with ITD1 (Figure 3C, D). In accordance with data from RT-qPCR experiments, the levels of expression of lipolysis genes (ATGL and HSL) were significantly elevated in BM adipocytes treated with RepSox compared with the levels in BM adipocytes treated with ITD1 (Online Supplementary Figure S3D). These results suggest that TGF $\beta$ RII is the major receptor that mediates GDF15 action on BM adipocytes.

To further verify the function of TGF $\beta$ RII on BM adipocytes, we knocked down TGF $\beta$ RII expression by shTGF $\beta$ RII lentivirus (Online Supplementary Figure S4A, B). As shown in Figure 3E and F, when TGF $\beta$ RII was knocked down in BM adipocytes, rhGDF15 did not significantly reduce the number and area of BM adipocytes. Accordingly, rhGDF15 did not significantly reduce the TRPV4 protein (Figure 3G). These results further confirmed that GDF15 regulates BM adipocyte remodeling by binding to TGF $\beta$ RII.

As an important signaling downstream pathway of the TGF $\beta$ family, GDF15 could not cause significant changes in $\mathrm{Smad} 2$ and Smad4 proteins in BM adipocytes (Figure $3 \mathrm{H}$ and Online Supplementary Figure S4C). Notably, when PI3K was blocked by PI3K-IN-1 (a PI3K inhibitor), rhGDF15 could not regulate AKT phosphorylation (Figure 3I), suggesting that GDF15 is involved in the remodeling of BM adipocytes by activating the PIBK/AKT pathway.

\section{The PI3K/AKT pathway inhibits the TRPV4 promoter F0XC1}

PIBK/AKT acts as a signaling pathway downstream of GDF15, which may affect the transcription or translation of TRPV4. We compared the expression of different transcription factors with or without rhGDF15 treatment by RNA-sequencing analysis. The results showed that the expression of several transcription factors decreased, including FOXC1, Spalt-like gene-2 (SALL2), and MYC associated factor X (MAX) (Figure 4A). Based on the criteria of a fold-change $>2.0$ and $P$-value $<0.05$, FOXC1 was identified as a transcription factor that was significantly changed in BM adipocytes after rhGDF15 treatment (Figure 4B). To investigate whether FOXC1 is responsible for GDF15 regulating TRPV4, we knocked down FOXC1 in BM adipocytes (Online Supplementary Figure $S 4 D, E)$. The results showed that the expression of TRPV4 at both the mRNA and protein levels was inhibited in FOXC1 knockdown adipocytes (Figure 4C, D), suggesting that GDF15 reduced the expression of TRPV4 by negatively regulating the transcription factor FOXC1.

To further demonstrate the link between FOXC1 and TRPV4, we used a FOXC1 antibody to pull DNA fragments containing FOXC1 and used RT-qPCR to detect the TRPV4 gene in the fragment. The results showed that the control group had the sequence of the TRPV4 gene and the amount of TRPV4 gene was correspondingly decreased after knocking down FOXC1 (Figure 4E). These data suggest that FOXC1 combines directly with TRPV4. As shown in Figure 4F, rhGDF15 downregulated the expression of FOXC1 and TRPV4 protein, but PI3K-IN-1 can block this process. Taken together, these results again demonstrate that GDF15 regulates TRPV4 channels through the PIBK/AKT pathway.

\section{TRPV4 plays an important role in bone marrow} adipocyte remodeling in acute myeloid leukemia mice

To better understand the role of TRPV4 in leukemia cell-induced BM adipocyte remodeling, we investigated the changes in number and size of $B M$ adipocytes with $4 \alpha \mathrm{PDD}$ in mice with leukemia. FBL-3 is a mouse-derived AML cell line, which can spontaneously induce leukemia. We first confirmed that FBL-3 cells secrete GDF15 (Online Supplementary Figure S5A). In vitro, FBL-3 cells co-cultured with BM adipocytes inhibited the expression of TRPV4 protein (Online Supplementary Figure S5B). Online Supplementary Figure S5C, D shows that FBL-3 cells reduced the number and area of $\mathrm{BM}$ adipocytes, while $4 \alpha \mathrm{PDD}$ could partly reverse this effect. Correspondingly, FBL-3 cells significantly promoted the expression of ATGL and HSL mRNA in BM adipocytes, but $4 \alpha \mathrm{PDD}$ 
A

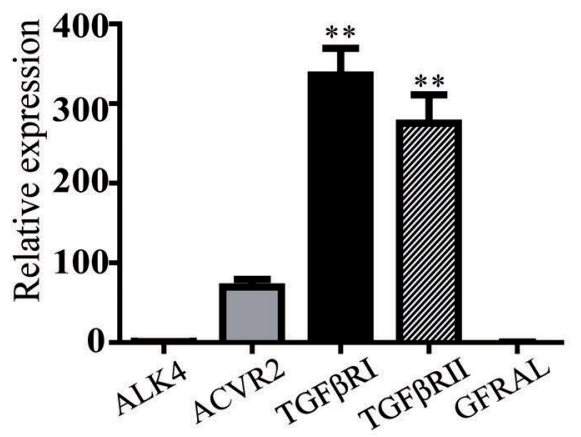

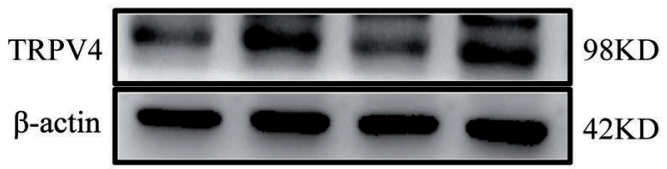

rhGDF15 + $\quad-\quad+\quad+$

RepSox - - $+t^{-}$

ITD1 - $\quad-\quad+\quad+$

C

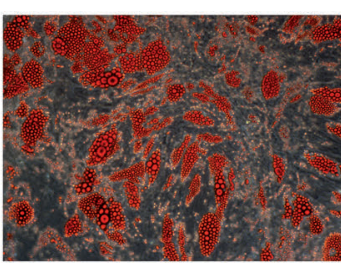

$\mathrm{Ctr}$

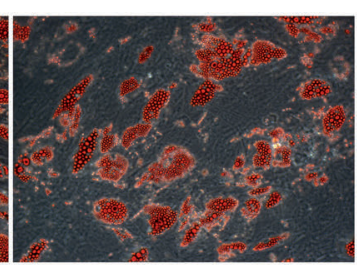

rhGDF15

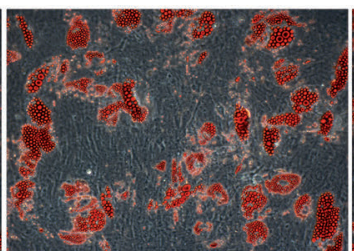

rhGDF15+RepSox

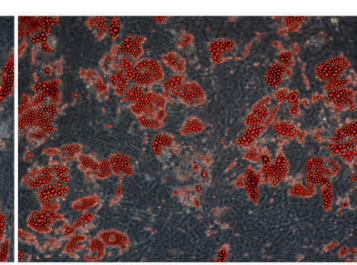

rhGDF15+ITD1
D

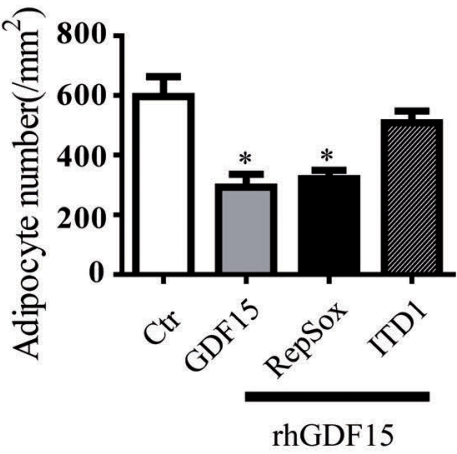

E

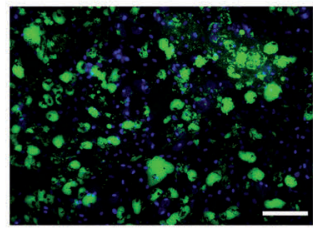

Ctr

$\mathrm{F}$

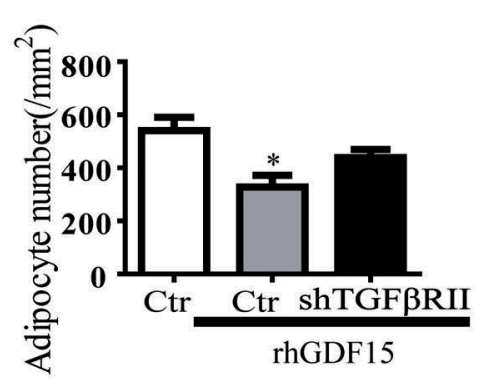

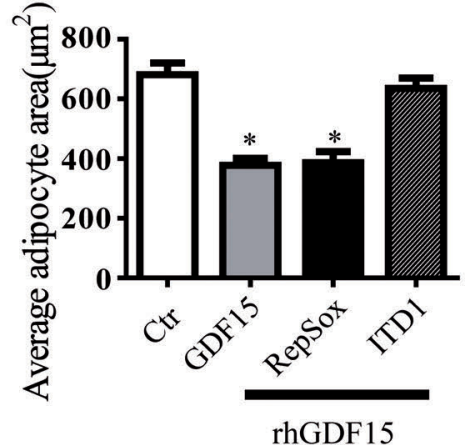

rhGDF15

G

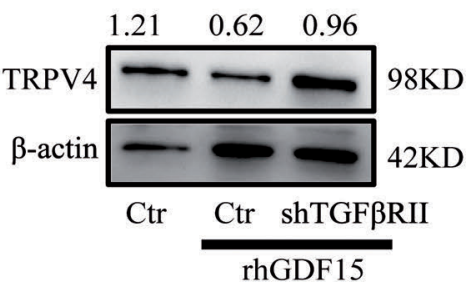

H

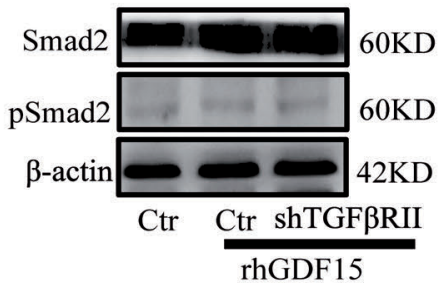

I

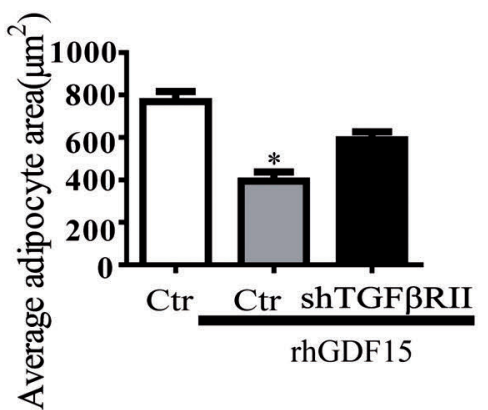

Figure 3. GDF15 activates the downstream genes PI3K and pAKT in bone marrow adipocyte remodeling. (A) Reverse transcriptase quantitative polymerase chain reaction (RT-qPCR) analysis of GDF15-related receptors in bone marrow (BM) adipocytes. (B) Western blot analysis of the expression of TRPV4 protein in BM adipocytes induced by recombinant human GDF15 (rhGDF15) after treatment with TGFßRI inhibitor (RepSox) or TGF $\beta$ RII inhibitor (ITD1) for 4 days. (C) Oil red O staining analysis of BM adipocytes induced by rhGDF15 after treatment with RepSox and ITD1 for 6 days. All images were at a magnification of $200 \times$. (D) The number and average area of BM adipocytes from the indicated groups were measured using Image-Pro-Plus 5.1. (E, F) BM adipocytes were infected with TGF $\beta$ RII-targeted shRNA (shTGFBRII) lentivirus for $48 \mathrm{~h}$ and then cultured with rhGDF15 for 6 days. Adipocytes were stained with Alexa Fluor 493/503-conjugated BODIPY. 4',6diamidino-2-phenylindole (DAPI) stained blue and lipid droplets showed green fluorescence. The number and average area of adipocytes from the indicated groups were measured using Image-Pro-Plus 5.1. The scale bar represents $50 \mu \mathrm{m}$. (G, H) BM adipocytes were infected with shTGF $\beta R I I$ lentivirus for $48 \mathrm{~h}$ and then cultured with rhGDF15 for 4 days. The levels of TRPV4, Smad2 and pSmad2 proteins were detected using western blot analysis. (I) BM adipocytes were treated with or without rhGDF15 and PI3K inhibitor (PI3K-IN-1, $2 \mu \mathrm{M}$ ) for 4 days. The levels of PI3K, AKT and pAKT proteins were detected using western blot analysis. $\beta$-actin protein was used as an internal control for the western blot analysis. Three independent experiments were performed. $* * P<0.01, * P<0.05$. 
could partly prevent this process (Online Supplementary Figure S5E). Additionally, $4 \alpha \mathrm{PDD}$ had no significant effect on the proliferation and apoptosis of FBL-3 cells, as determined by a CCK8 assay (Online Supplementary Figure S5F) and flow cytometry analysis (Online Supplementary Figure $S 5 G)$. Thus, these results suggest that this dose of $4 \alpha \mathrm{PDD}$ affects adipocytes, rather than directly affecting FBL-3 cells in the co-culture system.
A

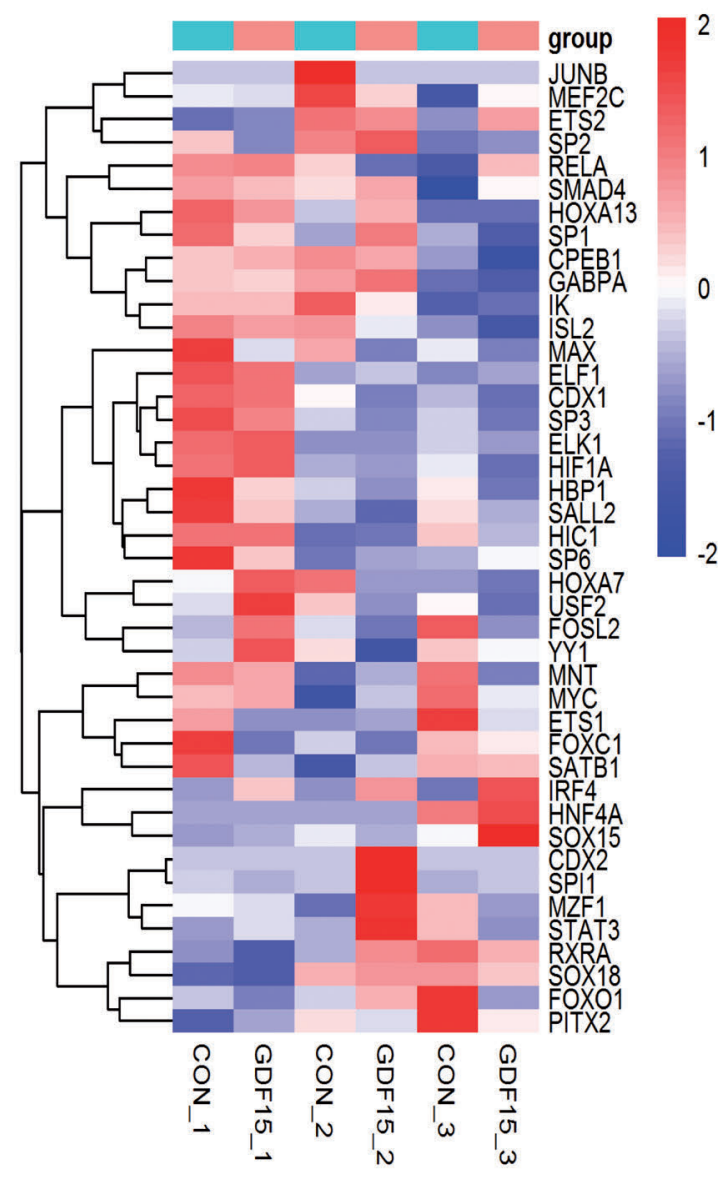

B

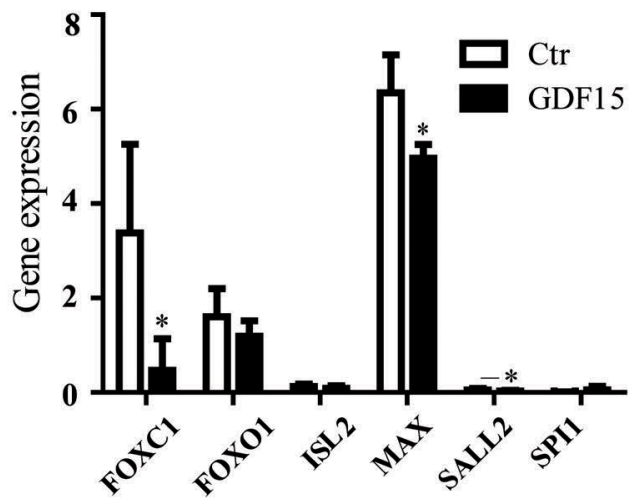

C

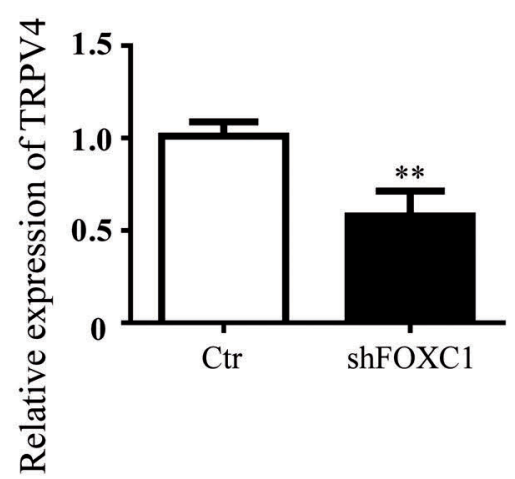

D

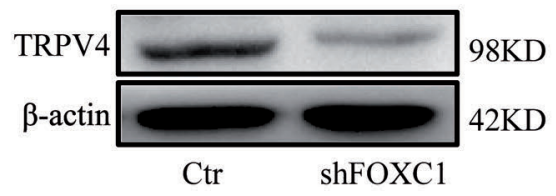

$\mathrm{E}$

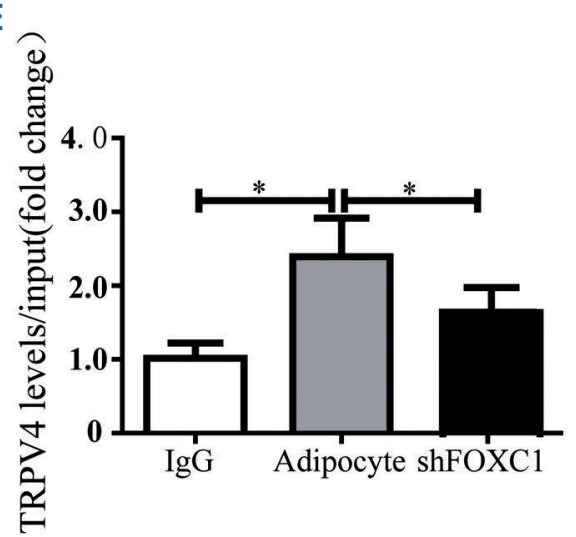

$\mathrm{F}$

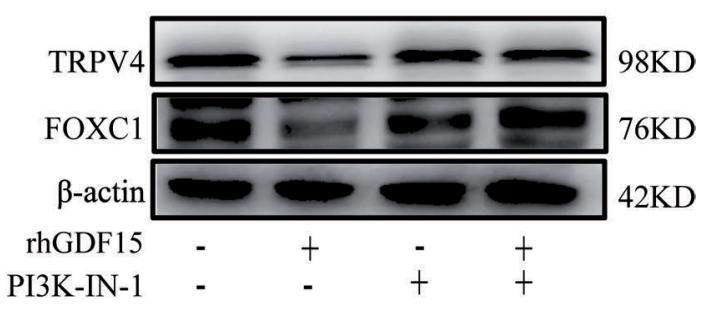

Figure 4. The PI3K/AKT pathway inhibits the TRPV4 promoter FOXC1. (A) Analysis of TRPV4 upstream transcription factor expression of bone marrow (BM) adipocytes treated with or without recombinant human GDF15 (rhGDF15) for 2 days by RNA sequencing). (B) Different expression of TRPV4-related transcription factor genes following rhGDF15 treatment for 2 days. (C) Reverse transcriptase quantitative polymerase chain reaction (RT-qPCR) was used to analyze TRPV4 mRNA level after treatment with FOXC1-targeted shRNA (shFOXC1) lentivirus for $48 \mathrm{~h}$. (D) Western blot was used to analyze the expression of TRPV4 protein after treatment with shFOXC1 lentivirus for $48 \mathrm{~h}$. (E) Chromatin immunoprecipitation-qPCR analysis of TRPV4 gene level in adipocytes with or without FOXC1 knockdown. (F) BM adipocytes were treated with or without rhGDF15 and PI3K inhibitor (PI3K-IN-1, $2 \mu \mathrm{M}$ ) for 4 days. The levels of FOXC1 and TRPV4 proteins were determined using western blot analysis. $\beta$ actin protein was used as an internal control for the western blot analysis. Three independent experiments were performed. $* * P<0.01, * P<0.05$. 
Considering that there were few adipocytes in the BM of C57BL/6 mice, we raised the mice with a high-fat diet to increase the number of adipocytes in the BM. BM adipocytes in obese mice are round or elliptical in shape
(Figure 5A). Immunohistochemical staining of perilipin1 protein in the $B M$ adipocytes showed that the $B M$ adipocytes stained yellow (Figure 5A). According to the pathogenic characteristics of FBL-3 cells, ${ }^{29} \mathrm{BM}$ samples
A

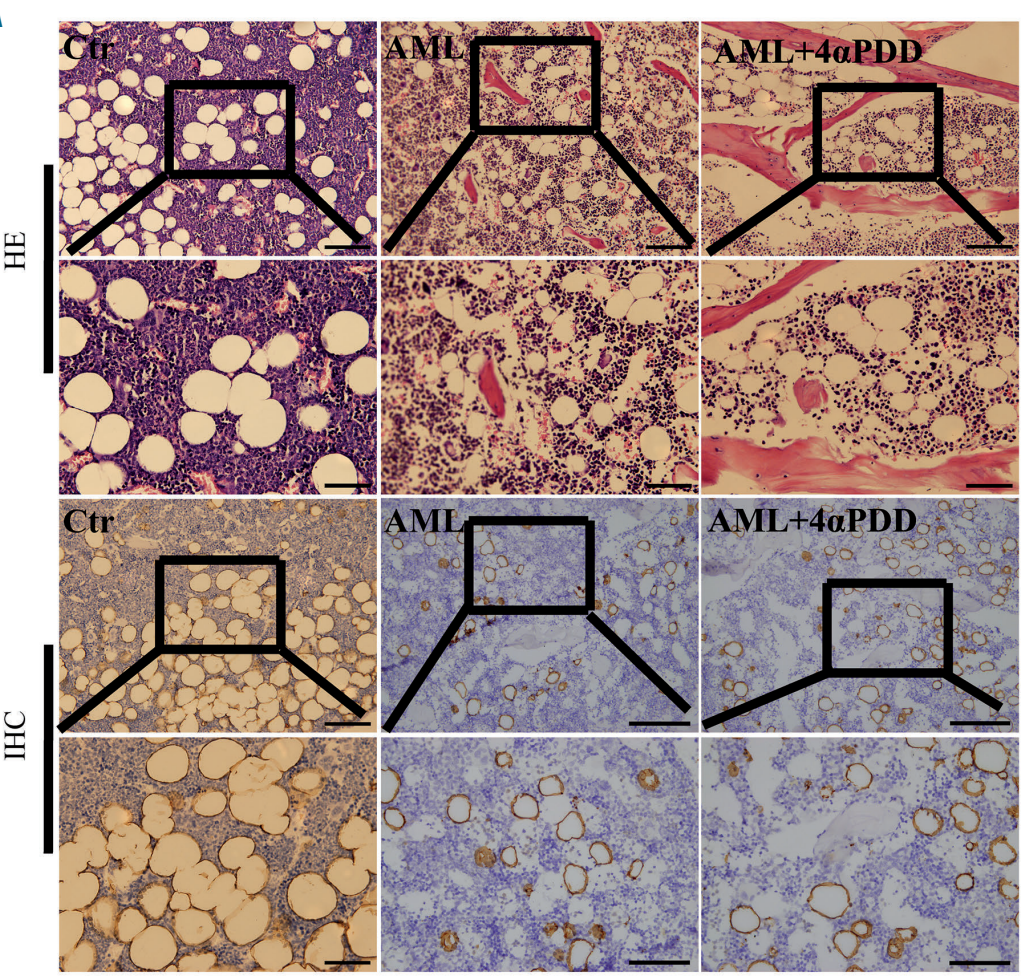

B
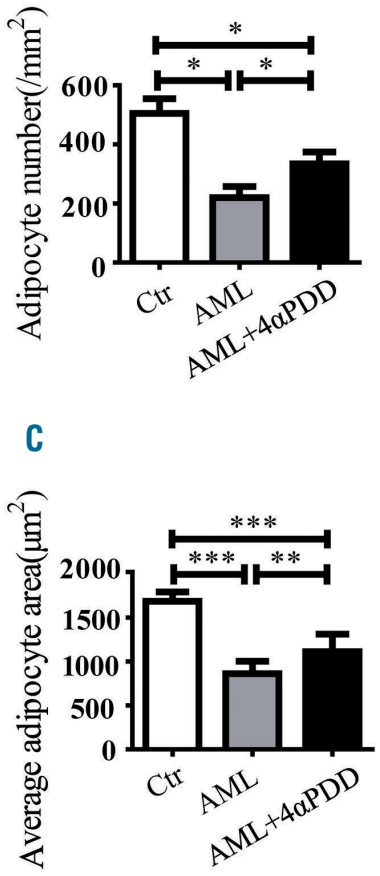

D

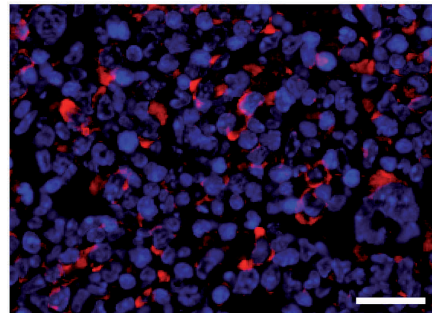

Ctr

$\mathrm{E}$

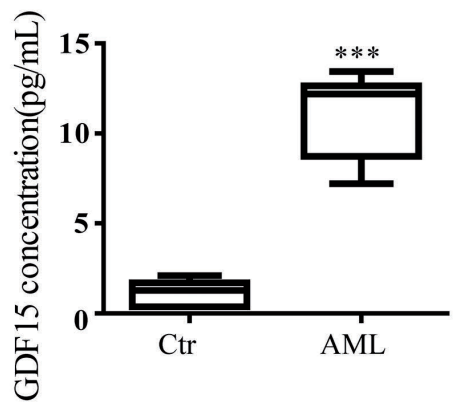

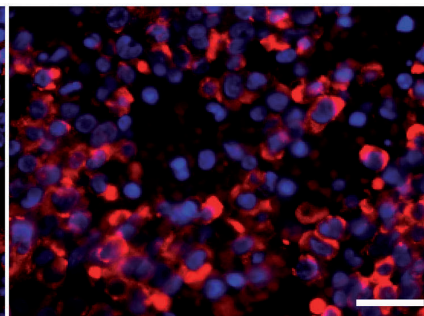

AML

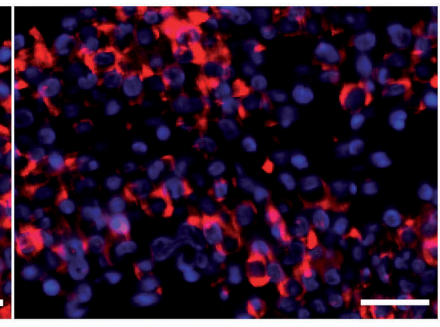

$\mathrm{AML}+4 \alpha \mathrm{PDD}$

$\mathrm{F}$

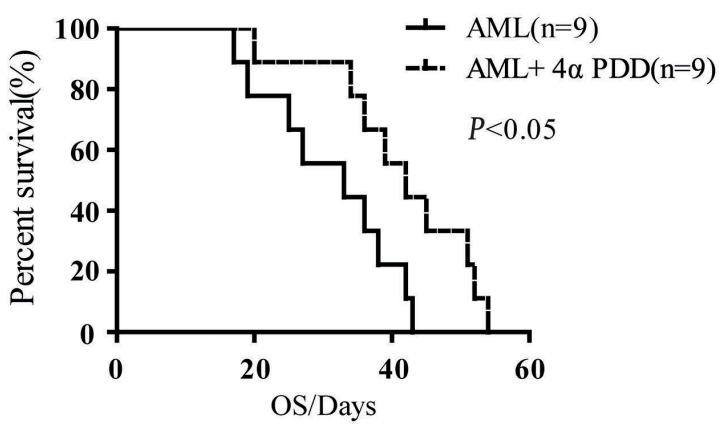

Figure 5. TRPV4 plays an important role in bone adipocyte remodeling in acute myeloid leukemia mice. (A) Hematoxylin \& eosin staining and immunohistochemica staining with perilipin1 antibody in bone marrow (BM) sections from the controls (Ctr, $n=5)$ and experimental mice, (mice with acute myeloid leukemia [AML], $n=5$; AML mice treated with $4 \alpha$-phorbol 12,13-didecanoate [ $4 \alpha P D D], n=5)$. Ten fields were analyzed for each mouse at $400 \times$ magnification. Three independent experiments were performed. Scale bars represent $50 \mu \mathrm{m}$ and $100 \mu \mathrm{m}$, respectively. (B, C) Adipocyte number and area in the controls, AML mice and AML mice treated with $4 \alpha$ PDD, quantitatively analyzed by Image-Pro-Plus 5.1. (D) Immunofluorescence was used to analyze the expression of CD117 in BM sections of the controls, AML mice and AML mice treated with 4 $\alpha$ PDD. 4',6-diamidino-2-phenylindole (DAPI) stained blue and CD117 showed red fluorescence. Scale bars represent $100 \mu$ m. (E) The GDF15 content of the BM supernatant in the controls and AML mice was analyzed by enzyme-linked immunosorbent assay. (F) Kaplan-Meier curves showing the overall survival rate of AML mice $(n=9)$ and AML mice treated with $4 \alpha \mathrm{PDD}(n=9)$. Three independent experiments were performed. $* * * P<0.001, * * P<0.01$, $\star P<0.05$. 
were taken 21 days after tail vein injection of FBL-3 cells. The shape of BM adipocytes did not change significantly, but the number and area decreased (Figure 5A). Further quantitative analysis showed that the number of $\mathrm{BM}$ adipocytes in AML mice $\left(219 \pm 37.7 / \mathrm{mm}^{2}\right)$ was lower than that in the controls $\left(505 \pm 49.7 / \mathrm{mm}^{2}\right)$ and AML mice treated with $4 \alpha \mathrm{PDD}\left(334.4 \pm 39.6 / \mathrm{mm}^{2}\right)$, but the number of BM adipocytes in AML mice treated with $4 \alpha \mathrm{PDD}$ was still lower than that in the controls (the $t$-test for any two groups: $P<0.05$ ) (Figure $5 \mathrm{~B})$. Similarly, the $\mathrm{BM}$ adipocyte area in mice with leukemia was $860.0 \pm 142.5 \mu \mathrm{m}^{2}$, which was smaller than that in the controls $\left(1686.4 \pm 106.7 \mu \mathrm{m}^{2}\right.$, $P<0.001)$. Meanwhile, the area of BM adipocytes in AML mice treated with $4 \alpha \mathrm{PDD}$ was $1111.8 \pm 201.5 \mu \mathrm{m}^{2}$, which was larger than that of the BM adipocytes in AML mice $(P<0.01)$, and did not return to normal $(P<0.001)$ (Figure $5 \mathrm{C})$.

We further found that there were more CD117 (a progenitor cell expression marker, red fluorescence)-positive cells in AML mice than in the AML mice treated with $4 \alpha P D D$ (Figure 5D). This may be due to the fact that GDF15 secreted by AML cells promotes lipolysis and is beneficial to the proliferation of leukemia cells, while $4 \alpha \mathrm{PDD}$ partly prevents lipolysis. In fact, the content of GDF15 in the BM supernatant was higher in AML mice than in the controls (Figure 5E), suggesting that GDF15 secreted by leukemia cells can promote lipolysis of BM adipocytes. Furthermore, we observed that treatment with $4 \alpha \mathrm{PDD}$ significantly extended overall survival of AML mice (Figure 5F). In brief, these results suggest that targeting TRPV4 in BM adipocytes can delay the progression of leukemia in mice.

\section{Discussion}

We have demonstrated a possible mechanism whereby TRPV4 mediates BM adipocyte responses to extracellular GDF15. Our data show that AML cells drive this remodeling process, at least in part, through TRPV4-dependent lipolysis in the adipocytes. Our previous reports linked increased levels of small adipocytes in BM to poor prognosis in AML patients, and revealed that GDF15 derived from leukemia cells remodels mature BM adipocytes into small adipocytes. ${ }^{5,12}$ Here, we found that GDF15 binds to its receptor TGF $\beta$ RII on BM adipocytes, which in turn activates downstream target genes, including PI3K and $A K T$. Subsequently, TRPV4 is inhibited via downregulation of its transcription factor FOXC1. These results suggest that GDF15 regulates TRPV4 through the above pathway, thereby promoting BM adipocyte remodeling (Online Supplementary Figure S6). This finding is consistent with several reports that TRPV4 acts as a volume receptor rather than an osmotic receptor..$^{30-32}$

There has been a report that TRPV4 is located on the cell membrane and acts as a calcium channel. ${ }^{33}$ Therefore, TRPV4 can regulate energy metabolism of peripheral white adipocytes by facilitating $\mathrm{Ca}^{2+}$ influx, which in turn stimulates the ERK1/2-dependent pathway. ${ }^{34}$ GDF15 inhibits the expression of TRPV4 in BM adipocytes, resulting in a decrease of $\mathrm{Ca}^{2+}$ influx (Online Supplementary Figure $S 3 A$ ) and an increase in pHSL protein (Online Supplementary Figure S2E) after BM adipocytes were treated with rhGDF15 for 4 days. It has been reported that reduced $\mathrm{Ca}^{2+}$ influx causes an increase in the expression of
pHSL, leading to lipolysis of adipocytes. ${ }^{17}$ Notably, the TRPV4 channel is a tetrameric complex formed by the same or similar monomeric subunits. ${ }^{35}$ Interestingly, cytosolic $\mathrm{N}$ - and C-terminal domains are involved in channel gating and mediating intracellular signaling, ${ }^{35,36}$ indicating that it is impossible for TRPV4 to interact directly with exogenous chemical factors. Hence, it would be interesting to examine how TRPV4 communicates with extracellular GDF15.

Given our findings that extracellular GDF15 inhibited the expression of TRPV4 in BM adipocytes, we speculated that GDF15 acts on BM adipocytes through TGF $\beta$ receptors. As a member of the TGF $\beta$ superfamily, GDF15 is known to interact with receptors of TGF $\beta$ members, such as TGF $\beta$ RI, TGF $\beta$ RII, ALK4 and ACVR2..$^{37-39}$ In addition, GDF15 has unique cognate receptors, such as GFRAL, which is mainly expressed in the central nervous system and, at low levels, in testicular tissue. ${ }^{39}$ Our data show that BM adipocytes express TGF $\beta$ receptors, but not the known unique GDF15 receptors. Moreover, TGF $\beta$ RII was shown to be associated with GDF15 activity on BM adipocytes (Figure $3 \mathrm{~B}-\mathrm{G}$ ). These experiments inform the first step of GDF15 acting on the adipocytes.

Our study further revealed that PIBK/AKT activation plays an essential role in driving GDF15 regulation of target genes in BM adipocytes. In fact, GDF15 induced the activation of Smad, a component of the classic anti-apoptosis pathway of cardiomyocytes which promotes the progression of lung cancer. ${ }^{40,41}$ However, we did not focus on the Smad pathway in this study because the activated Smad protein type is known to be determined by the TGF $\beta R I$ present in the ligand-bound signal complex. ${ }^{42}$ In fact, GDF15 did not affect the Smad signaling pathway in BM adipocytes, which is consistent with our results (Figure $3 \mathrm{H}$ and Online Supplementary Figure S4C).Taken together, our data, when interpreted in the context of previous reports, suggest that the PIBK/AKT pathway may be important for GDF15-induced remodeling of BM adipocytes.

Interestingly, we observed that PI3K/AKT activation downregulated the TRPV4-associated transcription factor FOXC1. FOXC1 is also a transcription factor of ITGA7 and FGFR4 in colorectal cancer, CXCR4 in endothelial cells, and FGF19 in ciliary body-derived cells. ${ }^{43-45}$ Moreover, the transcriptional function of FOXC1 has not been described previously for some pivotal adipogenic genes (FABP4, CEBPA and PPARG) and lipolytic genes (ATGL and HSL). Based on the knockdown of FOXC1 gene, the lipolytic gene in BM adipocytes increases (Online Supplementary Figure $S 4 F$ ), which confirmed that FOXC1 is important for regulating the metabolism of BM.

In addition, TRPV4 can be activated or inhibited by physical and chemical factors. When it comes to the matter of the size of cells, TRPV4 acts as a volume receptor rather than an osmotic receptor, ${ }^{30}$ suggesting that TRPV4 is involved in the regulation of cell volume. Previous studies have suggested that TRPV4 is an important inflammatory factor because TRPV4 levels are increased in inflamed tissues and activation of TRPV4 causes inflammation. ${ }^{46}$ This protein is also closely related to inflammation of white adipose tissue. ${ }^{33,47}$ However, the decreased expression of TRPV4 in leukemia-associated BM adipocytes implies that TRPV4 is not a major pro-inflammatory factor of leukemia-associated BM adipocytes. We conclude that downregulated TRPV4 preferentially pro- 
motes lipolysis in BM adipocytes, contributing to their remodeling into small adipocytes in the pathogenesis of AML.

Moreover, we also found that treatment with the TRPV4 agonist 4aPDD rescued BM adipocyte remodeling, which was correlated with increased survival in AML-bearing mice, supporting a crucial role of TRPV4 in the growth and progression of AML. Although there are unstained positive white circles in the BM of AML mice (Figure 5A), we suspect that these circles may represent an increase in blood vessels or sinuses in the BM of these animals. Leukemia cells can promote angiogenesis, which in turn contributes to the proliferation of leukemia cells. ${ }^{48}$ Of course, it cannot be excluded that a few adipocytes were not stained positively. Many studies have also reported the effect of circulating factors in obese animals on leukemia. ${ }^{49,50}$ However, in our study, both experimental groups and control groups were obese mice, and their basic background was the same. This should, therefore, have allowed us to control for the impact of the circulat- ing factors, which sould not have been responsible for observed differences in mouse survival and other variables. Future studies in vivo are needed to validate the specific modulatory role of GDF15 on TRPV4.

In conclusion, leukemia cells activate a transcriptional network that includes GDF15-related-PI3K/AKT activation and subsequent TRPV4 downregulation, which promotes BM adipocyte remodeling. The morphological adaptation of BM adipocytes and the modulation of lipolysis may represent a novel strategy for the treatment of hematologic malignancies, especially in elderly patients, whose aging and increased adiposity of the BM microenvironment reduce the efficacy of cytotoxic chemotherapy.

\section{Acknowledgments}

The work was supported by grants from the National Natural Science Foundation of China (grant n. 81870132), Science and Technology Commission of Shanghai Municipality (grant n.18DZ2293500) and Shanghai Sailing Program (grant $n$. 18YF1419100).

\section{References}

1. Asada N. Regulation of malignant hematopoiesis by bone marrow microenvironment. Front Oncol. 2018;8:119.

2. Schepers K, Campbell TB, Passegue E. Normal and leukemic stem cell niches: insights and therapeutic opportunities. Cell Stem Cell. 2015;16(3):254-267.

3. Tabe Y, Yamamoto S, Saitoh $\mathrm{K}$, et al. Bone marrow adipocytes facilitate fatty acid oxidation activating AMPK and a transcriptional network supporting survival of acute monocytic leukemia cells. Cancer Res. 2017;77(6):1453-1464.

4. Lee MKS, Al-Sharea A, Dragoljevic D, Murphy AJ. Hand of FATe: lipid metabolism in hematopoietic stem cells. Curr Opin Lipidol. 2018;29(3):240-245.

5. Lu W, Weng W, Zhu Q, et al. Small bone marrow adipocytes predict poor prognosis in acute myeloid leukemia. Haematologica. 2018;103(1):e21-e24.

6. Shafat MS, Oellerich T, Mohr S, et al. Leukemic blasts program bone marrow adipocytes to generate a protumoral microenvironment. Blood. 2017;129(10): 1320-1332.

7. Choe SS, Huh JY, Hwang IJ, Kim JI, Kim JB. Adipose tissue remodeling: its role in energy metabolism and metabolic disorders. Front Endocrinol (Lausanne). 2016;7:30.

8. Bochet L, Lehuede C, Dauvillier S, et al. Adipocyte-derived fibroblasts promote tumor progression and contribute to the desmoplastic reaction in breast cancer. Cancer Res. 2013;73(18):5657-5668.

9. Herroon MK, Rajagurubandara E, Hardaway AL, et al. Bone marrow adipocytes promote tumor growth in bone via FABP4-dependent mechanisms. Oncotarget. 2013;4 (11):2108-2123.

10. Dirat B, Bochet L, Dabek M, et al. Cancerassociated adipocytes exhibit an activated phenotype and contribute to breast cancer invasion. Cancer Res. 2011;71(7):2455-2465.

11. Fraczak E, Olbromski M, Piotrowska A, et al. Bone marrow adipocytes in haematological malignancies. Acta Histochem. 2018;120(1):22-27
12. Lu W, Wan Y, Li ZQ, et al. Growth differentiation factor 15 contributes to marrow adipocyte remodeling in response to the growth of leukemic cells. J Exp Clin Cancer Res. 2018;37(1):66.

13. Lu JM, Wang CY, Hu CL, Fang YJ, Mei YA. GDF-15 enhances intracellular $\mathrm{Ca} 2+$ by increasing Cav1.3 expression in rat cerebellar granule neurons. Biochem J. 2016;473 (13):1895-1904

14. Zemel M, Thompson W, Milstead A, Morris $\mathrm{K}$, Campbell P. Calcium and dairy acceleration of weight and fat loss during energy restriction in obese adults. Obes Res. 2004;12(4):582-590

15. Shi H, Dirienzo D, Zemel MB. Effects of dietary calcium on adipocyte lipid metabolism and body weight regulation in energy restricted aP2-agouti transgenic mice. FASEB J. 2001;15(2):291-293

16. Hashimoto R, Katoh Y, Miyamoto Y, et al. High extracellular $\mathrm{Ca} 2+$ enhances the adipocyte accumulation of bone marrow stromal cells through a decrease in cAMP. Cell Calcium. 2017;67:74-80

17. Xue B, Greenberg AG, Kraemer FB, Zemel $M B$. Mechanism of intracellular calcium $([\mathrm{Ca} 2+] \mathrm{i})$ inhibition of lipolysis in human adipocytes. FASEB J. 2001;15(13):2527-2529.

18. Kim JH,Mynatt RL, Moore JW, Woychik RP, Moustaid N, Zemel MB. The effects of calcium channel blockade on agouti-induced obesity. FASEB J. 1996;10(14):1646-1652.

19. He YH, Zhang HO, Teng JH, Huang LN, Li $\mathrm{Y}$, Sun $\mathrm{CH}$. Involvement of calcium-sensing receptor in inhibition of lipolysis through intracellular cAMP and calcium pathways in human adipocytes. Biochem Biophys Res Commun. 2011;404(1):393-399.

20. Alexander R, Kerby A, Aubdool AA, et al. 4alpha-phorbol 12,13-didecanoate activates cultured mouse dorsal root ganglia neurons independently of TRPV4. Br J Pharmacol. 2013;168(3):761-772.

21. Sergeev IN, Li S, Ho CT, Rawson NE, Dushenkov S. Polymethoxyflavones activate $\mathrm{Ca} 2+$-dependent apoptotic targets in adipocytes. J Agric Food Chem. 2009;57(13): 5771-5776.

22. Camell CD, Sander J, Spadaro O, et al. Inflammasome-driven catecholamine catab- olism in macrophages blunts lipolysis during ageing. Nature. 2017;550(7674):119-123

23. Li JY, Gong L, Liu SZ, et al. Adipose HuR protects against diet-induced obesity and insulin resistance. Nat Commun. 2019;10(1) 2375.

24. Wang F, Ren XF, Chen Z, et al. The N-terminal His-tag affects the triglyceride lipase activity of hormone-sensitive lipase in testis. J Cell Biochem. 2019;120(8):13706-13716.

25. Eguchi J, Wang X, Yu ST, et al. Transcriptional control of adipose lipid handling by IRF4. Cell Metab. 2011,13(3):249259.

26. Liu DD, Lu JM, Zhao OR, Hu CL, Mei YA Growth differentiation factor-15 promotes glutamate release in medial prefrontal cortex of mice through upregulation of T-type calcium channels. Sci Rep. 2016;6:28653.

27. Xu MY, Pang QQ, Xu SQ, et al. Hypoxiainducible factor- $1 \alpha$ activates transforming growth factor- $\beta 1 /$ Smad signaling and increases collagen deposition in dermal fibroblasts. Oncotarget. 2017;9(3):31883197

28. Bansal V, De D, An J, et al. Chemical induced conversion of mouse fibroblasts and human adipose-derived stem cells into skeletal muscle-like cells. Biomaterials. 2019;193:30-46

29. Herberman RB, Holden HT, Ting CC, Lavrin DL, Kirchner H. Cell-mediated immunity to leukemia virus- and tumor-associated antigens in mice. Cancer Res. 1976;36:615-621.

30. Toft-Bertelsen TL, Krizaj D, MacAulay N. When size matters: transient receptor potential vanilloid 4 channel as a volume-sensor rather than an osmo-sensor. J Physiol. 2017;595(11):3287-3302.

31. Rübenhagen $R$, Rönsch $H$, Jung $H$, Krämer $\mathrm{R}$, Morbach S. Osmosensor and osmoregulator properties of the betaine carrier BetP from Corynebacterium glutamicum in proteoliposomes. J Biol Chem. 2000;275(2):735741.

32. Heide T, Stuart MC, Poolman B. On the osmotic signal and osmosensing mechanism of an $\mathrm{ABC}$ transport system for glycine betaine. EMBO J. 2001;20(24):7022-7032.

33. Ramsey IS, Delling M, Clapham DE. An introduction to TRP channels. Annu Rev 
Physiol. 2006;68:619-647

34. Ye L, Kleiner S, Wu J, et al. TRPV4 is a regulator of adipose oxidative metabolism, inflammation, and energy homeostasis. Cell. 2012;51(1):96-110

35. Venkatachalam K, Montell C. TRP channels. Annu Rev Biochem. 2007;76:387-417.

36. Taberner FJ, Fernández-Ballester G, Fernández-Carvajal A, Ferrer-Montiel A. TRP channels interaction with lipids and its implications in disease. Biochim Biophys Acta. 2015;1848(9):1818-1827.

37. Artz A, Butz S, Vestweber D. GDF-15 inhibits integrin activation and mouse neutrophil recruitment through the ALK-5/TGFbetaRII heterodimer. Blood. 2016;128(4): 529-541.

38. Mullican SE, Lin-Schmidt $X$, Chin CN, et al. GFRAL is the receptor for GDF15 and the ligand promotes weight loss in mice and nonhuman primates. Nat Med. 2017;23(10): 1150-1157.

39. Yang L, Chang CC, Sun Z, et al. GFRAL is the receptor for GDF15 and is required for the anti-obesity effects of the ligand. Nat Med. 2017;23(10):1158-1166.
40. Heger J, Schiegnitz E, von Waldthausen D, Anwar MM, Piper HM, Euler G. Growth differentiation factor 15 acts anti-apoptotic and pro-hypertrophic in adult cardiomyocytes. J Cell Physiol. 2010;224(1): 120-126.

41. Lu Y, Ma J, Li Y, et al. CDP138 silencing inhibits TGF-beta/Smad signaling to impair radioresistance and metastasis via GDF15 in lung cancer. Cell Death Dis. 2017;8(9): e3036.

42. Olsen OE, Skjaervik A, Stordal BF, Sundan A, Holien T. TGF-beta contamination of purified recombinant GDF15. PLoS One. 2017;12(11)e0187349.

43. Liu J, Zhang Z, Li X, et al. Forkhead box C1 promotes colorectal cancer metastasis through transactivating ITGA7 and FGFR4 expression. Oncogene. 2018;37(41):5477. 5491.

44. Hayashi H, Kume T. Forkhead transcription factors regulate expression of the chemokine receptor CXCR4 in endothelial cells and CXCL12-induced cell migration. Biochem Biophys Res Commun. 2008;367(3):584-589. 45. Tamimi Y, Skarie JM, Footz T, Berry FB, Link
BA, Walter MA. FGF19 is a target for FOXC1 regulation in ciliary body-derived cells. Hum Mol Genet. 2006;15(21):3229 3240.

46. Ryskamp DA, Iuso A, Krizaj D. TRPV4 links inflammatory signaling and neuroglial swelling. Channels (Austin). 2015;9(2):7072 .

47. Sanchez IC, Rivera RA, Munoz LV. TRPV4 channels in human white adipocytes: electrophysiological characterization and regulation by insulin. J Cell Physiol. 2016;231(4): 954-963.

48. Hussong JW, Rodgers GM, Shami PJ Evidence of increased angiogenesis in patients with acute myeloid leukemia. Blood. 2000;95(1):309-313.

49. Lu Z, Xie J, Wu G, et al. Fasting selectively blocks development of acute lymphoblastic leukemia via leptin-receptor upregulation Nat Med. 2017;23(1):79-90

50. Yan F, Shen N, Pang JX, et al. Fatty acid-binding protein FABP4 mechanistically links obesity with aggressive AML by enhancing aberrant DNA methylation in AML cells Leukemia. 2017;31(6):1434-1442. 